\title{
On the Shadow Simplex Method for Curved Polyhedra
}

\author{
Daniel Dadush $^{1}$ and Nicolai Hähnle ${ }^{2}$ \\ 1 Centrum Wiskunde \& Informatica, The Netherlands \\ dadush@cwi.nl \\ 2 Universität Bonn, Germany \\ haehnle@or.uni-bonn.de
}

\begin{abstract}
We study the simplex method over polyhedra satisfying certain "discrete curvature" lower bounds, which enforce that the boundary always meets vertices at sharp angles. Motivated by linear programs with totally unimodular constraint matrices, recent results of Bonifas et al. (SOCG 2012), Brunsch and Röglin (ICALP 2013), and Eisenbrand and Vempala (2014) have improved our understanding of such polyhedra.

We develop a new type of dual analysis of the shadow simplex method which provides a clean and powerful tool for improving all previously mentioned results. Our methods are inspired by the recent work of Bonifas and the first named author [8], who analyzed a remarkably similar process as part of an algorithm for the Closest Vector Problem with Preprocessing.

For our first result, we obtain a constructive diameter bound of $O\left(\frac{n^{2}}{\delta} \ln \frac{n}{\delta}\right)$ for $n$-dimensional polyhedra with curvature parameter $\delta \in(0,1]$. For the class of polyhedra arising from totally unimodular constraint matrices, this implies a bound of $O\left(n^{3} \ln n\right)$. For linear optimization, given an initial feasible vertex, we show that an optimal vertex can be found using an expected $O\left(\frac{n^{3}}{\delta} \ln \frac{n}{\delta}\right)$ simplex pivots, each requiring $O(m n)$ time to compute. An initial feasible solution can be found using $O\left(\frac{m n^{3}}{\delta} \ln \frac{n}{\delta}\right)$ pivot steps.
\end{abstract}

1998 ACM Subject Classification G.1.6 Optimization

Keywords and phrases Optimization, Linear Programming, Simplex Method, Diameter of Polyhedra

Digital Object Identifier 10.4230/LIPIcs.SOCG.2015.345

\section{Introduction}

The simplex method is one of the most important methods for solving linear programs (LPs), that is, optimization problems of the form $\max \{\langle\mathbf{c}, \mathbf{x}\rangle: \mathbf{x} \in P\}$ where $P$ is a polyhedron defined by linear constraints. Starting from an initial vertex $\mathbf{v}$, a simplex algorithm provides a rule for moving from vertex to vertex along edges of the graph or 1-skeleton of $P$ until an optimal vertex w (or an unbounded ray) is found.

A long standing open question is whether there exists a polynomial-time simplex algorithm for LPs. The first obstacle in proving the existence (or non-existence) of such a method is the following fundamental question:

- Question 1. Given any two vertices $\mathbf{v}, \mathbf{w}$ of a polyhedron $P$, what is the best possible bound on the length of the shortest path between them, as a function of the dimension $n$ and the number of constraints $m$ ?

cc) (i) Daniel Dadush and Nicolai Hähnle;

licensed under Creative Commons License CC-BY

31st International Symposium on Computational Geometry (SoCG'15).

Editors: Lars Arge and János Pach; pp. 345-359

Leibniz International Proceedings in Informatics

LIPICS Schloss Dagstuhl - Leibniz-Zentrum für Informatik, Dagstuhl Publishing, Germany 
The polynomial Hirsch conjecture posits that the diameter of the graph of a polyhedron is bounded by a polynomial in $m$ and $n$. The best known general upper bounds are however much larger. Barnette [3] and Larman [15] proved a bound of $O\left(2^{n} m\right)$, and Todd [20] recently proved a bound of $(m-n)^{\log n}$, slightly improving an earlier bound of Kalai and Kleitman [13, 14]. The original Hirsch conjecture, which posited a bound of $m-n$, was recently disproved for polytopes (i.e. bounded polyhedra) by Santos $[18,16]$, who gave a lower bound of $(1+\varepsilon) m$ (only slightly violating the conjectured bound).

Given the difficulty of the general question, much research has been aimed at bounding the diameter of special classes of polyhedra. For example, polynomial bounds have been given for $0 / 1$ polytopes [17], transportation polytopes [2, 6, 10], and flag polytopes [1].

Another important class, which has recently received much attention and is directly related to this work, are polyhedra whose constraint matrices are "well-conditioned". Dyer and Frieze [11] showed that the diameter of totally unimodular polyhedra - i.e. having integer constraint matrices with all subdeterminants in $\{0, \pm 1\}$ - is bounded by $O\left(n^{16} m^{3}(\log n m)^{3}\right)$. Their work also contains a polynomial time randomized simplex algorithm that solves linear programs over totally unimodular polyhedra.

The diameter bound of Dyer and Frieze was both generalized and improved in the work of Bonifas et al. [4]. They showed that polyhedra with integer constraint matrices and all subdeterminants bounded by $\Delta$ have diameter $O\left(\Delta^{2} n^{4} \log (n \Delta)\right)$ if they are unbounded and $O\left(\Delta^{2} n^{3.5} \log (n \Delta)\right)$ if they are bounded. Their proof used certain expansion properties of the polyhedral graph and was non-constructive.

In an attempt to make the bound of [4] constructive, Brunsch and Röglin [7] showed that given any two vertices $\mathbf{v}, \mathbf{w}$ on such a polyhedron $P$, a path between them of length $O\left(m \Delta^{4} n^{4}\right)$ (note the dependence on $m$ ) can be constructed using the shadow simplex method. In fact, they give a more general bound based on the so-called $\delta$-distance property of the constraint matrix, which measures how "well spread" the rows of the constraint matrix are ${ }^{1}$. Using this parameter they give a bound of $O\left(m n^{2} / \delta^{2}\right)$ on the length of the constructed path, and recover the previous bound by the relationship $\delta \geq 1 /\left(n \Delta^{2}\right)$.

Most recently, Eisenbrand and Vempala [12] provided a different approach to making the Bonifas et al. [4] result constructive, which more closely resembles the random walk approach of Dyer and Frieze and also extends to optimization. When the constraint matrix satisfies the $\delta$-distance property, they show that given an initial vertex and objective, an optimal vertex can be computed using poly $(n, 1 / \delta)$ random walk steps (no dependence on $m$ ). Furthermore, an initial feasible vertex can be computed using $m$ calls to their optimization algorithm over subsets of the original constraints.

\section{Results}

Building and improving upon the works of Bonifas et al. [4], Brunsch and Röglin [7], and Vempala and Eisenbrand [12], we give an improved (constructive) diameter bound and simplex algorithm for polyhedra satisfying the $\delta$-distance and other related properties. We also make improvements in the treatment of unbounded polyhedra and degeneracy. All our results are based on a new variant and analysis of the shadow simplex method.

We now introduce the "discrete curvature measures" we use along with the corresponding results. We list these measures in order of increasing strength. In the next section, we

1 We note that this measure is already implicit in [4] and that the diameter bound factors through it. 
shall explain our variant of the shadow simplex method and compare it with previous implementations.

Let $P=\left\{\mathbf{x} \in \mathbb{R}^{n}: A \mathbf{x} \leq \mathbf{b}\right\}, A \in \mathbb{R}^{m \times n}, \mathbf{b} \in \mathbb{R}^{n}$ be a pointed polyhedron ( $A$ has full column rank $\Leftrightarrow P$ has vertices). For a vertex $\mathbf{v}$ of $P$, the normal cone at $\mathbf{v}$ is $N_{\mathbf{v}}=\left\{\sum_{i \in I_{\mathbf{v}}} \lambda_{i} \mathbf{a}_{i}: \lambda_{i} \geq 0, i \in I_{\mathbf{v}}\right\}$, where $I_{\mathbf{v}}=\left\{i \in[m]:\left\langle\mathbf{a}_{i}, \mathbf{v}\right\rangle=\mathbf{b}_{i}\right\}$ is the set of tight constraints. Equivalently, $N_{\mathbf{v}}$ is the set of all linear objective functions whose maximum over $P$ is attained at $\mathbf{v} . N_{\mathbf{v}}$ is simplicial (non-degenerate) if it is generated by a basis of $A$, that is, if exactly $n$ linearly independent constraints of $P$ are tight at $\mathbf{v}$. The normal fan of $P$ is the collection of all the vertex normal cones, and the support of the normal fan $N(P)$ is their union. A polyhedron is simple (or non-degenerate) if all its vertex normal cones are simplicial.

- Definition 2 ( $\tau$-wide Polyhedra). We say that a cone $C$ is $\tau$-wide if it contains a Euclidean ball of radius $\tau$ centered on the unit sphere. We define a polyhedron $P$ to have a $\tau$-wide normal fan (or simply $P$ to be $\tau$-wide) if every vertex normal cone is $\tau$-wide.

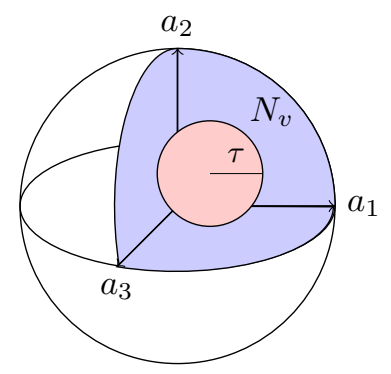

Roughly speaking, having a $\tau$-wide normal fan enforces that facets always intersect at "sharp angles" (i.e. angle bounded away from $\pi$ ). In particular, for any vertex $\mathbf{v}$ of $P$, the angle between any two rays emanating from $\mathbf{v}$ and (non-trivially) passing through $P$ is at most $\pi-2 \tau$. Hence one can interpret this condition as a discrete form of curvature for polyhedra. We now state our diameter bound for $\tau$-wide polyhedra.

- Theorem 3 (Diameter Bound, see Theorem 11). Let $P \subseteq \mathbb{R}^{n}$ be an n-dimensional pointed polyhedron having a $\tau$-wide normal fan. Then the graph of $P$ has diameter bounded by $8 n / \tau(1+\ln (1 / \tau))$. Furthermore, a path of this expected length can be constructed via the shadow simplex method.

Restricting to $n$-dimensional polyhedra with subdeterminants bounded by $\Delta$, using the relation $\tau \geq 1 /(n \Delta)^{2}$ (for a proof, see the full version of the paper) we achieve a bound of $O\left(n^{3} \Delta^{2} \ln (n \Delta)\right)$, improving on the existential bounds of Bonifas et al. [4]. When $A$ is totally unimodular, the bound becomes $O\left(n^{3} \ln n\right)$. In contrast to [4], we note that our bound (and proof) is the same for polytopes and unbounded polyhedra.

While our bound is constructive - we follow a shadow simplex path - it is in general only efficiently implementable when the polyhedron is simple. In the presence of degeneracy, we note that computing a single edge of the path is essentially as hard as solving linear programming. Furthermore, standard techniques for removing degeneracy, such as the perturbation or lexicographic method, may unfortunately introduce a large number of extra simplex pivots.

Interestingly, our diameter bound can take advantage of degeneracy in situations where it makes the normal cones wider. While degeneracy does not occur for "generic polyhedra", it is very common for combinatorial polytopes. Furthermore, it can occur in ways that are 
useful to our diameter bound. For example, we remark that using degeneracy one can prove that the normal fan of the perfect matching polytope is $\Omega(1 / \sqrt{|E|})$-wide [9].

To solve linear optimization problems via the shadow simplex method, we will need more than a wide normal fan. In fact, we will have different requirements for the two phases of the simplex algorithm: Phase 1, which finds an initial feasible vertex, will require more than Phase 2, which finds an optimal vertex with respect to the objective starting from a feasible vertex.

- Definition 4 ( $\delta$-distance property). A set of linearly independent vectors $\mathbf{v}_{1}, \ldots, \mathbf{v}_{k} \in \mathbb{R}^{n}$ satisfy the $\delta$-distance property if for every $i \in[k]$, the vector $\mathbf{v}_{i}$ is at Euclidean distance at least $\delta\left\|\mathbf{v}_{i}\right\|$ from the span of $\left\{\mathbf{v}_{j}: j \in[k] \backslash\{i\}\right\}$.

For a polyhedron $P=\left\{\mathbf{x} \in \mathbb{R}^{n}: A \mathbf{x} \leq \mathbf{b}\right\}$, we define $P$ to satisfy the local $\delta$-distance property if every feasible basis of $A$, i.e. the rows of $A$ defining a vertex of $P$, satisfies the $\delta$-distance property.

We say that a set of vectors $\mathbf{v}_{1}, \ldots, \mathbf{v}_{m} \in \mathbb{R}^{n}$ satisfy the global $\delta$-distance property if every linearly independent subset satisfies the $\delta$-distance property. We say that a matrix $A \in \mathbb{R}^{m \times n}$ satisfies the global $\delta$-distance property if its row vectors do.

- Lemma 5. Let $\mathbf{v}_{1}, \ldots, \mathbf{v}_{n} \in \mathbb{S}^{n-1}$ be a basis satisfying the $\delta$-distance property. Then cone $\left(\mathbf{v}_{1}, \ldots, \mathbf{v}_{n}\right)$ is $\delta / n$-wide.

Proof. See full paper.

The definitions differ in strength mainly based on the sets of bases to which they apply. The local $\delta$-distance property is stronger than the $\tau$-wide property for $\tau=\delta / n$, because it implies that all triangulations of the normal fan are $\tau$-wide. ${ }^{2}$ The global property is stronger than the local property since it applies also to infeasible bases, which allows one to control the geometry of polyhedra related to $P$, such as polyhedra obtained by removing a subset of constraints, which will be needed for Phase 1.

We now state our main result for Phase 2 simplex.

- Theorem 6 (Optimization via Shadow Simplex, see Theorem 20). Let $P=\left\{\mathbf{x} \in \mathbb{R}^{n}: A \mathbf{x} \leq \mathbf{b}\right\}$ be an $n$-dimensional polytope with $m$ constraints satisfying the local $\delta$-distance property. Then, given an objective $\mathbf{c} \in \mathbb{R}^{n}$ and a vertex $\mathbf{v}$ of $P$, an optimal vertex can be computed using an expected $O\left(\left(n^{3} / \delta\right) \ln (n / \delta)\right)$ shadow simplex pivots, where each pivot requires $O(m n)$ arithmetic operations.

Our Phase 2 algorithm above is faster than the algorithms in [7, 12] and relies on a weaker assumption than [12]. The $\mathbf{v}, \mathbf{w}$ path finding algorithm of Brunsch and Röglin [7] is in fact a special case of the above, since we can choose $\mathbf{c}$ to be any objective maximized at w. Comparing to the Phase 2 algorithm of Eisenbrand and Vempala [12], we require only the local $\delta$-distance property instead of the global one. Whether one could rely only on the local property was left as open question in [12], which we resolve in the affirmative.

A small technical caveat is that as stated, the algorithm requires knowledge of $\delta$. Since $\delta \leq$ 1 , we can always guess a number $\delta^{\prime} \leq \delta \leq 2 \delta^{\prime}$ by trying $O(\ln 1 / \delta)$ different values, incurring an $O(\ln 1 / \delta)$ factor increase in running time (overestimating $\delta$ only affects correctness, not runtime). For simplicity, we shall henceforth assume that $\delta$ is known.

2 However, the $\tau$-wide property is weaker even when all normal cones are simplicial: a 2-dimensional cone of inner angle close to $\pi$ is almost 1-wide, but satisfies $\delta$-distance only for $\delta$ close to 0 . 
A more important caveat is that the above algorithm requires that $P$ be a polytope (i.e. bounded). This restriction is due to the fact that we can only generate the randomness required for our bounds efficiently (that is, without solving a general LP) when the support of the normal fan equals $\mathbb{R}^{n}$.

The unbounded setting can be reduced to the bounded setting, in the standard way, by adding one or more constraints to make $P$ bounded while not cutting off any of its vertices.

- Definition 7. Let $P=\left\{\mathbf{x} \in \mathbb{R}^{n}: A \mathbf{x} \leq \mathbf{b}\right\}$ be a pointed polyhedron. Then a polytope $P^{\prime}=\left\{\mathbf{x} \in \mathbb{R}^{n}: A \mathbf{x} \leq \mathbf{b}, A^{\prime} \mathbf{x} \leq \mathbf{b}^{\prime}\right\}$ is $L P$ equivalent to $P$ if every vertex $\mathbf{v} \in P$ satisfies $\left\langle\mathbf{a}_{i}^{\prime}, \mathbf{v}\right\rangle<\mathbf{b}_{i}^{\prime}$ for all $i$; in particular, $\mathbf{v}$ is a vertex of $P^{\prime}$.

Given an optimal vertex $\mathbf{v}$ of $P^{\prime}$ as above, one can easily check whether $\mathbf{v}$ is a vertex of $P$. If it is not, the original LP must be unbounded. In general, however, adding constraints to $P$ happens at the expense of a degraded $\delta$. In particular, the standard reduction of adding a large box constraint can degrade $\delta$ arbitrarily, hence the constraints must be added with care. We state the guarantees we can achieve below.

- Lemma 8 (Removing Unboundedness, see full paper). Let $P=\left\{\mathbf{x} \in \mathbb{R}^{n}: A \mathbf{x} \leq \mathbf{b}\right\}$ be an $n$-dimensional pointed polyhedron with $m$ constraints. Let $\mathbf{a}_{1}, \ldots, \mathbf{a}_{m}$ denote the rows of $A$ and $b_{\max }=\max _{i \in[m]}\left|\mathbf{b}_{i}\right| /\left\|\mathbf{a}_{i}\right\|$.

1. Assume that $P$ satisfies the local $\delta$-distance property and that $I \subseteq[m],|I|=n$, indexes the rows of a feasible basis. Letting $\mathbf{w}=-1 / n \sum_{i \in I} \mathbf{a}_{i} /\left\|\mathbf{a}_{i}\right\|$, we have that

$$
P^{\prime}=\left\{\mathbf{x} \in \mathbb{R}^{n}: A \mathbf{x} \leq \mathbf{b}, \quad\langle\mathbf{w}, \mathbf{x}\rangle \leq n b_{\max } / \delta\right\},
$$

is a polytope that is LP equivalent to $P$ and satisfies the local $\delta^{2} /(2 n)$-distance property. 2. Assume that $A$ satisfies the global $\delta$-distance property. Then

$$
P^{\prime}=\left\{\mathbf{x} \in \mathbb{R}^{n}:-n\left\|\mathbf{a}_{i}\right\| b_{\max } / \delta-1 \leq\left\langle\mathbf{a}_{i}, \mathbf{x}\right\rangle \leq \mathbf{b}_{i}, \quad \forall i \in[m]\right\}
$$

is a polytope that is LP equivalent to $P$ and satisfies the global $\delta$-distance property.

Finally, we use standard techniques for reducing feasibility to Phase 2 type optimization. As this generally requires pivoting over infeasible bases, we will require global instead of local properties here. Interestingly, for LPs with bounded subdeterminants, we get that the number of simplex pivots is completely independent of the number of constraints.

- Theorem 9 (Feasibility via Shadow Simplex, see full paper). Let $P=\left\{\mathbf{x} \in \mathbb{R}^{n}: A \mathbf{x} \leq b\right\}$ be an $n$-dimensional polyhedron whose constraint matrix has full column rank and satisfies the global $\delta$-distance property. Then a feasible solution to $P$ can be computed using an expected $O\left(\left(m n^{3} / \delta\right) \ln (n / \delta)\right)$ shadow simplex pivots. Furthermore, if $A$ is integral and has subdeterminants bounded by $\Delta$, a feasible solution can be computed using an expected $\left.O\left(n^{5} \Delta^{2} \ln (n \Delta)\right)\right)$ shadow simplex pivots.

\section{Shadow Simplex Method}

Our main technical contribution is a new analysis and variant of the shadow simplex method, which utilizes (rather unexpectedly) an approach developed in [8] for navigating over the Voronoi graph of a Euclidean lattice (see related work section).

The shadow simplex has been at the heart of many theoretical attempts to explain the surprising efficiency of the simplex method in practice. It has been shown to give polynomial bounds for the simplex method over random and smoothed linear programs $[5,19,21]$. As 
mentioned above, Brunsch and Röglin [7] already showed that it yields short paths for the polyhedra we consider here.

At a high level, the shadow simplex over a polyhedron $P$ works as follows. Given an initial objective function $\mathbf{c}$, a vertex $\mathbf{v}$ of $P$ which maximizes this objective, and a target objective function $\mathbf{d}$, the shadow simplex interpolates between the objective functions $\mathbf{c}$ and $\mathbf{d}$ and performs a pivot step whenever the optimal vertex changes (hence the alternative name parametric simplex method referring to the parameterization $\mathbf{c}(\lambda)=(1-\lambda) \mathbf{c}+\lambda \mathbf{d}$ of the objective function, where $\lambda$ grows from 0 to 1 over the course of the algorithm).

Traditionally, this method is understood and analyzed with a primal interpretation: The polyhedron $P$ is orthogonally projected onto the 2-dimensional plane spanned by $\mathbf{c}$ and $\mathbf{d}$ (hence the term "shadow"), and the algorithm is understood in terms of the boundary of the projection $P^{\prime}$. The optimal vertices for $\mathbf{c}$ and $\mathbf{d}$ project to the boundary of $P^{\prime}$, and as long as $\mathbf{c}$ and $\mathbf{d}$ are in sufficiently general position, edges of $P^{\prime}$ lift to edges of $P$ so that the boundary can be followed efficiently by an algorithm that performs simplex pivots in the original space. The number of pivot steps is then typically bounded in terms of the lengths of edges or in terms of angles between edges of $P^{\prime}$.

Our analysis is substantially different and based on a dual perspective: The shadow simplex method follows the line segment $[\mathbf{c}, \mathbf{d}]$ through the normal fan of $P$, pivoting whenever the segment crosses into a different $n$-dimensional normal cone. We express the number of crossings, that is, the number of intersections between $[\mathbf{c}, \mathbf{d}]$ and the facets of the normal fan of $P$, in terms of certain surface area measures of translates of the normal fan. The bounds we obtain on the number of intersections are stated below.

- Theorem 10 (Intersection bounds, see Lemmas 22 and 25). Let $\mathcal{T}=\left(C_{1}, \ldots, C_{k}\right)$ be a partition of a cone $\Sigma$ into polyhedral $\tau$-wide cones. Let $\mathbf{c}, \mathbf{d} \in \mathbb{R}^{n}$ and let $X \in \mathbb{R}^{n}$ be exponentially distributed on $\Sigma$ (see Section 3.1).

1. The expected number of facets hit by the shifted line segment $[\mathbf{c}+X, \mathbf{d}+X]$ satisfies

$$
\mathbb{E}[\mid \partial \mathcal{T} \cap[\mathbf{c}+X, \mathbf{d}+X] \|] \leq \frac{\|\mathbf{d}-\mathbf{c}\|}{\tau} .
$$

2. Let $\alpha \in(0,1)$. Then

$$
\mathbb{E}[|\partial \mathcal{T} \cap[\mathbf{c}+\alpha X, \mathbf{c}+X]|] \leq \frac{2 n}{\tau} \ln \frac{1}{\alpha} .
$$

To achieve the above bounds, the main idea is to relate the probability that the above random line segments pass through a normal cone to the probability that the associated perturbation vector lands in the cone (or some joint shift). Under the $\tau$-wideness condition, we can in fact uniformly upper bound these proportionality factors. Since the jointly shifted normal cones are all disjoint, we can deduce the desired bounds from the fact that the sum of their measures is $\leq 1$.

We compose these bounds in a way that also departs from the classic template by using three consecutive shadow simplex paths instead of just one. For given vertices $\mathbf{v}$ and $\mathbf{w}$ we first pick objectives $\mathbf{c}$ and $\mathbf{d}$ that are "deep inside" the respective normal cones. From here, we sample an exponentially distributed perturbation vector $X$ and traverse three paths through the normal fan in sequence:

$$
\mathbf{c} \stackrel{(a)}{\longrightarrow} \mathbf{c}+X \stackrel{(b)}{\longrightarrow} \mathbf{d}+X \stackrel{(c)}{\longrightarrow} \mathbf{d}
$$

The perturbation $X$ will be quite large and hence almost always large enough to push $\mathbf{c}$ and d away from their normal cones. Indeed, the high level intuition behind our path is that in 
order to avoid unusually long paths from $\mathbf{c}$ to $\mathbf{d}$, we first travel to a "random intermediate location".

We note that in phases $(a)$ and $(c)$, randomness is only used to perturb one of the objectives. As far as we are aware, this paper provides the first successful analysis of the shadow simplex path in this setting. Furthermore, this extension is crucial to achieving our improved diameter bound. Previous algorithms were constrained to random perturbations that kept $\mathbf{c}$ and $\mathbf{d}$ inside their respective normal cones, making the amount of randomness they could take advantage of much smaller.

We now use the bounds from Theorem 10 to derive the diameter bound.

- Theorem 11. Let $P \subseteq \mathbb{R}^{n}$ be a pointed full dimensional polyhedron with $\tau$-wide normal cones. Then $P$ has diameter bounded by $\frac{8 n}{\tau}(1+\ln 1 / \tau)$.

Proof. Let $\mathbf{v}_{1}, \mathbf{v}_{2}$ be vertices of $P$ with normal cones $N_{\mathbf{v}_{1}}, N_{\mathbf{v}_{2}}$. Let $\mathbf{c}_{1}, \mathbf{c}_{2} \in \mathbb{S}^{n-1}$ satisfy $\mathbf{c}_{i}+\tau \mathcal{B}_{2}^{n} \subseteq N_{\mathbf{v}_{i}}, i \in\{1,2\}$. Let $\Sigma=N(P)$ denote the support of the normal fan of $P$, and let $X$ be exponentially distributed over $\Sigma$.

We will construct a path from $\mathbf{v}_{1}$ to $\mathbf{v}_{2}$ by following the sequence of vertices optimizing the objectives in the segments $\left[s \mathbf{c}_{1}, s \mathbf{c}_{1}+X\right],\left[s \mathbf{c}_{1}+X, s \mathbf{c}_{2}+X\right],\left[s \mathbf{c}_{2}+X, s \mathbf{c}_{2}\right]$, where $s>0$ is a scalar to be chosen later. We will condition on the event that $\|X\| \leq 2 n$. Since $\mathbb{E}[\|X\|]=n$ (see Lemma 13), by Markov's inequality this occurs with probablity at least $1 / 2$. Under this event, by $\tau$-wideness, we will not pivot in the segments $\left[s \mathbf{c}_{1}, s \mathbf{c}_{1}+\frac{s \tau}{2 n} X\right]$ and $\left[s \mathbf{c}_{2}+\frac{s \tau}{2 n} X, s \mathbf{c}_{2}\right]$. Using Theorem 10, the number of pivots along the segments $\left[s \mathbf{c}_{1}+\frac{s \tau}{2 n} X, s \mathbf{c}_{1}+X\right],\left[s \mathbf{c}_{1}+\right.$ $\left.X, s \mathbf{c}_{2}+X\right],\left[s \mathbf{c}_{2}+X, s \mathbf{c}_{2}+\frac{s \tau}{2 n} X\right]$, is bounded by

$$
\frac{\left(\frac{s\left\|\mathbf{c}_{2}-\mathbf{c}_{1}\right\|}{\tau}+\frac{4 n}{\tau} \ln \left(\frac{2 n}{s \tau}\right)\right)}{\operatorname{Pr}[\|X\| \leq 2 n]} \leq 2\left(\frac{s\left\|\mathbf{c}_{2}-\mathbf{c}_{1}\right\|}{\tau}+\frac{4 n}{\tau} \ln \left(\frac{2 n}{s \tau}\right)\right) .
$$

Setting $s=\frac{4 n}{\left\|\mathbf{c}_{2}-\mathbf{c}_{1}\right\|}$, the above bound becomes

$$
\frac{8 n}{\tau}\left(1+\ln \left(\frac{\left\|\mathbf{c}_{2}-\mathbf{c}_{1}\right\|}{2 \tau}\right)\right) \leq \frac{8 n}{\tau}\left(1+\ln \frac{1}{\tau}\right), \quad \text { as needed. }
$$

\section{Related Work}

In a surprising connection, we borrow techniques developed in a recent work of Bonifas and the first named author [8] for a totally different purpose, namely, for solving the Closest Vector Problem with Preprocessing on Euclidean lattices. In [8], a 3-step "perturbed" line path was analyzed to navigate over the Voronoi graph of the lattice, where lattice points are connected if their associated Voronoi cells touch in a facet.

In the current work, we show a strikingly close analogy between analyzing the number of intersections of a random straight line path with a Voronoi tiling of space and the intersections of a shadow simplex path with the normal fan of a polyhedron. This unexpected connection makes us hopeful that these ideas may have even broader applicability.

\section{$3 \quad$ Notation and Definitions}

For vectors $\mathbf{x}, \mathbf{y} \in \mathbb{R}^{n}$, we let $\langle\mathbf{x}, \mathbf{y}\rangle=\sum_{i=1}^{n} \mathbf{x}_{i} \mathbf{y}_{i}$ denote their inner product. We let $\|\mathbf{x}\|=\sqrt{\langle\mathbf{x}, \mathbf{x}\rangle}$ denote the Euclidean norm, $\mathcal{B}_{2}^{n}=\left\{\mathbf{x} \in \mathbb{R}^{n}:\|\mathbf{x}\| \leq 1\right\}$ the unit ball, and $\mathbb{S}^{n-1}=\partial \mathcal{B}_{2}^{n}$ the unit sphere. We denote the linear span of a set $A \subseteq \mathbb{R}^{n}$ by $\operatorname{span}(A)$. We use the notation $\mathrm{I}[\mathbf{x} \in A]$ for the indicator of $A$, that is $\mathrm{I}[\mathbf{x} \in A]$ is 1 if $\mathbf{x} \in A$ and 
0 otherwise. For a set of scalars $S \subseteq \mathbb{R}$, we write $S A=\{s \mathbf{a}: s \in S, \mathbf{a} \in A\}$. For two sets $A, B \subseteq \mathbb{R}^{n}$, we define their Minkowski sum $A+B=\{\mathbf{a}+\mathbf{b}: \mathbf{a} \in A, \mathbf{b} \in B\}$. We let $d(A, B)=\inf \{\|\mathbf{x}-\mathbf{y}\|: \mathbf{x} \in A, \mathbf{y} \in B\}$, denote the Euclidean distance between $A$ and $B$. For vectors $\mathbf{a}, \mathbf{b} \in \mathbb{R}^{n}$ we write $[\mathbf{a}, \mathbf{b}]$ for the closed line segment and $[\mathbf{a}, \mathbf{b})$ for the half-open line segment from $\mathbf{a}$ to $\mathbf{b}$.

- Definition 12 (Cone). A cone $\Sigma \subseteq \mathbb{R}^{n}$ satisfies the following three properties:

$0,0 \in \Sigma$.

$-\mathbf{x}+\mathbf{y} \in \Sigma$ if $\mathbf{x}$ and $\mathbf{y}$ are in $\Sigma$.

$-\lambda \mathbf{x} \in \Sigma$ if $\mathbf{x} \in \Sigma$ and $\lambda \geq 0$.

For vectors $\mathbf{y}_{1}, \ldots, \mathbf{y}_{k} \in \mathbb{R}^{n}$, we define the closed cone they generate as

$$
\operatorname{cone}\left(\mathbf{y}_{1}, \ldots, \mathbf{y}_{k}\right)=\left\{\sum_{i=1}^{m} \lambda_{i} \mathbf{y}_{i}: \lambda_{i} \geq 0, i \in[m]\right\} .
$$

A cone is polyhedral if it can be generated by a finite number of vectors, and is simplicial if the generators are linearly independent. By convention, we let cone $(\emptyset)=\mathbf{0}$. A simplicial cone has the $\delta$-distance property if its extreme rays satisfy the $\delta$-distance property. ${ }^{3}$

The faces of a convex set $K \subseteq \mathbb{R}^{n}$ are its subsets of the form $F=\{\mathbf{x} \in K:\langle\mathbf{a}, \mathbf{x}\rangle=\beta\}$ where $\mathbf{a} \in \mathbb{R}^{n}$ and $\beta \in \mathbb{R}$ satisfy $\langle\mathbf{a}, \mathbf{x}\rangle \leq \beta$ for all $\mathbf{x} \in K$. Faces of co-dimension 1 are called facets. For a simplicial cone $C$, we note that its non-empty faces are exactly all the subcones generated by any subset of the generators of $C$.

A set of cones $\mathcal{T}=\left\{C_{1}, \ldots, C_{k}\right\}$ is an $n$-dimensional cone partition if:

- Each $C_{i} \subseteq \mathbb{R}^{n}, i \in[k]$, is a closed $n$-dimensional cone.

- Any two cones $C_{i}, C_{j}, i \neq j$, meet in a shared face.

- The support of $\mathcal{T}, \sup (\mathcal{T}) \stackrel{\text { def }}{=} \cup_{i \in[k]} C_{i}$, is a closed cone.

We say that $F$ is a face of $\mathcal{T}$ if it is a face of one of its contained cones. A cone partition $\mathcal{T}$ is $\tau$-wide if every $C_{i}$ is $\tau$-wide. It is simplicial if every $C_{i}$ is simplicial. In this case, we also call $\mathcal{T}$ a cone triangulation. A cone triangulation satisfies the local $\delta$-distance property if every $C_{i}$ satisfies it. We define the boundary of $\mathcal{T}, \partial \mathcal{T}=\cup_{i=1}^{k} \partial C_{i}$. We say that a cone triangulation $\mathcal{T}$ triangulates a cone partition $\mathcal{P}$ if $\mathcal{T}$ and $\mathcal{P}$ have the same support and every cone $C \in \mathcal{T}$ is generated by a subset of the extreme rays of some cone of $\mathcal{P}$. This means that $\mathcal{T}$ partitions ("refines") every cone of $\mathcal{P}$ into simplicial cones.

\subsection{Exponential distribution}

We say that a random variable $X \in \mathbb{R}^{n}$ is exponentially distributed on a cone $\Sigma$ if

$$
\operatorname{Pr}[X \in S]=\int_{S} \zeta_{\Sigma}(\mathbf{x}) \mathrm{d} \mathbf{x}
$$

for every measurable $S \subseteq \mathbb{R}^{n}$, where $\zeta_{\Sigma}(\mathbf{x})=c_{\Sigma} e^{-\|\mathbf{x}\|} \mathrm{I}[\mathbf{x} \in \Sigma]$. A standard computation, which we include for completeness, yields the normalizing constant and the expected norm.

- Lemma 13. The normalizing constant is $c_{\Sigma}^{-1}=n ! \operatorname{vol}_{n}\left(\mathcal{B}_{2}^{n} \cap \Sigma\right)$. For $X$ exponentially distributed on $\Sigma$, we have that $\mathbb{E}[\|X\|]=n$.

Proof. See full paper.

3 The $\delta$-distance property is invariant under scaling, so the choice of generators of the extreme rays is irrelevant. 


\section{Optimization}

While bounding the number of intersections of line segments $[\mathbf{c}, \mathbf{d}]$ with the facets of the normal fan of $P=\left\{\mathbf{x} \in \mathbb{R}^{n}: A \mathbf{x} \leq \mathbf{b}\right\}$ is sufficient to obtain existential bounds on the diameter of $P$, we also need to be able to efficiently compute the corresponding pivots to obtain efficient algorithms. The following summarizes the required results, the technical details of which are found in the full version of the paper.

- Theorem 14 (Shadow Simplex). Let $P=\left\{\mathbf{x} \in \mathbb{R}^{n}: A \mathbf{x} \leq \mathbf{b}\right\}$ be pointed, $\mathbf{c}, \mathbf{d} \in \mathbb{R}^{n}$, and $B$ an optimal basis for $\mathbf{c}$. If every intersection of $[\mathbf{c}, \mathbf{d})$ with a facet $F$ of a cone spanned by a feasible basis of $P$ lies in the relative interior of $F$, the Shadow Simplex can be used to compute an optimal basis for $\mathbf{d}$ in $O\left(m n^{2}+N m n\right)$ arithmetic operations, where $N$ is the number of intersections of $[\mathbf{c}, \mathbf{d}]$ with some triangulation $\mathcal{T}$ of the normal fan of $P$, where $\mathcal{T}$ contains the cone spanned by the initial basis $B$.

As explained in Section 2, we want to follow segments $[\mathbf{c}, \mathbf{c}+X],[\mathbf{c}+X, \mathbf{d}+X],[\mathbf{d}+X, \mathbf{d}]$ in the normal fan. Our intersection bounds from Theorem 10 are not quite sufficient to bound the number of steps on the first and last segments entirely. This is easily dealt with for the first segment, because we can control the initial objective function c so that it lies deep in the initial normal cone.

For the final segment, we follow the approach of Eisenbrand and Vempala [12], who showed that if $A$ satisfies the global $\delta$-distance property, then an optimal facet for $\mathbf{d}$ can be derived from a basis that is optimal for some $\tilde{\mathbf{d}}$ with $\|\mathbf{d}-\tilde{\mathbf{d}}\| \leq \frac{\delta}{n}$. Recursion can then be used on a problem of reduced dimension to move from $\tilde{\mathbf{d}}$ to $\mathbf{d}$. We strengthen their result (thereby answering a question left open by [12]) and show that the local $\delta$-distance property is sufficient to get the same result as long as $\|\mathbf{d}-\tilde{\mathbf{d}}\| \leq \frac{\delta}{n^{2}} .{ }^{4}$

- Lemma 15. Let $\mathbf{x}_{1}, \ldots, \mathbf{x}_{m} \in \mathbb{S}^{n-1}$ be a set of vectors. Then the following are equivalent:

1. $\mathbf{x}_{1}, \ldots, \mathbf{x}_{m}$ satisfy the $\delta$-distance property.

2. $\forall I \subseteq[m]$ for which $\left\{\mathbf{x}_{i}: i \in I\right\}$ are linearly independent and $\forall\left(a_{i} \in \mathbb{R}: i \in I\right)$

$$
\left\|\sum_{i \in I} a_{i} \mathbf{x}_{i}\right\| \geq \delta \max _{i \in I}\left|a_{i}\right|
$$

Proof. See full paper.

- Definition 16. Let $F$ be a face of a cone triangulation $\mathcal{T}$ and let $\mathbf{x}$ be a vector in the support of $\mathcal{T}$. Let $G=\operatorname{cone}\left(\mathbf{x}_{1}, \ldots, \mathbf{x}_{k}\right),\left\|\mathbf{x}_{i}\right\|=1$, be the minimal face of $\mathcal{T}$ that contains $\mathbf{x}$ and consider the unique conic combination $\mathbf{x}=\lambda_{1} \mathbf{x}_{1}+\cdots+\lambda_{k} \mathbf{x}_{k}$. We define

$$
\alpha_{F}(\mathbf{x}):=\sum_{i: \mathbf{x}_{i} \notin F} \lambda_{i}
$$

In particular, $\alpha_{F}(\mathbf{x}) \geq 1$ if $\mathbf{x}$ is a unit vector and the minimal face containing it is disjoint from $F$, and $\alpha_{F}(\mathbf{x})=0$ if $\mathbf{x} \in F$.

- Lemma 17. Let $F$ be a cone of an $n$-dimensional cone triangulation $\mathcal{T}$ satisfying the local $\delta$-distance property. Let $\mathbf{x}$ be a point in the support of $\mathcal{T}$. Then $d(\mathbf{x}, F) \geq \alpha_{F}(\mathbf{x}) \cdot \frac{\delta}{n}$.

\footnotetext{
${ }^{4}$ In the final bound, the loss of a factor $n$ here disappears inside a logarithm.
} 
Proof. Let $\mathbf{y} \in F$ be the (unique) point with $d(\mathbf{x}, \mathbf{y})=d(\mathbf{x}, F)$. Note that by convexity, the segment $[\mathbf{x}, \mathbf{y}]$ is contained in the support of $\mathcal{T}$. By considering the cones of $\mathcal{T}$ that contain points on the segment $[\mathbf{x}, \mathbf{y}]$, we obtain a sequence of points

$$
\mathbf{x}=\mathbf{x}_{0}, \mathbf{x}_{1}, \ldots, \mathbf{x}_{r}=\mathbf{y}
$$

on the segment $[\mathbf{x}, \mathbf{y}]$ and (full-dimensional) cones $G_{1}, \ldots, G_{r}$ such that

$$
G_{i} \cap[\mathbf{x}, \mathbf{y}]=\left[\mathbf{x}_{i-1}, \mathbf{x}_{i}\right] .
$$

Since $\alpha_{F}(\mathbf{y})=0$, the result of the lemma follows immediately from the claim that

$$
d\left(\mathbf{x}_{i-1}, \mathbf{x}_{i}\right) \geq\left|\alpha_{F}\left(\mathbf{x}_{i-1}\right)-\alpha_{F}\left(\mathbf{x}_{i}\right)\right| \cdot \frac{\delta}{n},
$$

which we will now prove.

Fix some $G_{i}=\operatorname{cone}\left(\mathbf{y}_{1}, \ldots, \mathbf{y}_{n}\right)$. By relabelling, we may assume that cone $\left(\mathbf{y}_{1}, \ldots, \mathbf{y}_{k}\right)=$ $G_{i} \cap F$ (since $G_{i}$ and $F$ are both faces of $\mathcal{T}$ ), for some $0 \leq k \leq n$ (if $k=0$ then $G_{i} \cap F=\{\mathbf{0}\}$ ).

For every $\mathbf{z} \in G_{i}$, the minimal cone containing $\mathbf{z}$ is a face of $G_{i}$. Therefore, using the unique conic combination $\mathbf{z}=\sum_{i=1} \lambda_{i} \mathbf{y}_{i}$, we have that $\alpha_{F}(\mathbf{z})=\sum_{k<i \leq n} \lambda_{i}$.

Writing $\mathbf{x}_{i-1}=\sum_{i=1}^{n} a_{i} \mathbf{y}_{i}$ and $\mathbf{x}_{i}=\sum_{i=1}^{n} b_{i} \mathbf{y}_{i}$, by Lemma 15 we have that

$$
\begin{aligned}
d\left(\mathbf{x}_{i-1}, \mathbf{x}_{i}\right) & \geq \delta \max _{1 \leq i \leq n}\left|a_{i}-b_{i}\right| \geq \delta \max _{k<i \leq n}\left|a_{i}-b_{i}\right| \geq \frac{\delta}{n} \sum_{k<i \leq n}\left|a_{i}-b_{i}\right| \\
& \geq \frac{\delta}{n}\left|\alpha_{F}\left(\mathbf{x}_{i-1}\right)-\alpha_{F}\left(\mathbf{x}_{i}\right)\right|,
\end{aligned}
$$

which completes the proof of the claim.

- Lemma 18. Let $F$ be a cone of a triangulation $\mathcal{T}$ satisfying the local $\delta$-distance property and let $\mathbf{x}$ be a point in the support of $\mathcal{T}$ with $d(\mathbf{x}, F) \leq \frac{\delta}{n^{2}}$. Let $G=\operatorname{cone}\left(\mathbf{x}_{1}, \ldots, \mathbf{x}_{n}\right)$, $\left\|\mathbf{x}_{i}\right\|=1$, be a cone of $\mathcal{T}$ containing $\mathbf{x}$ and let

$$
\mathbf{x}=\lambda_{1} \mathbf{x}_{1}+\cdots+\lambda_{n} \mathbf{x}_{n}
$$

be the corresponding conic combination. Then for every $i \in[n]$ with $\lambda_{i}>\frac{1}{n}$ one has $\mathbf{x}_{i} \in F$.

Proof. Suppose there is some $i$ with $\lambda_{i}>\frac{1}{n}$ and $\mathbf{x}_{i} \notin F$. Then $\alpha_{F}(\mathbf{x})>\frac{1}{n}$ and by Lemma 17 we get $d(\mathbf{x}, F)>\frac{\delta}{n^{2}}$, which is a contradiction.

For the recursion on a facet, we let $\pi_{i}(\mathbf{x}):=\mathbf{x}-\frac{\left\langle\mathbf{x}, \mathbf{a}_{i}\right\rangle}{\left\langle\mathbf{a}_{i}, \mathbf{a}_{i}\right\rangle} \mathbf{a}_{i}$ be the orthogonal projection onto the subspace orthogonal to $\mathbf{a}_{i}$ and we let $F_{i}$ be the facet of $P$ defined by $\left\langle\mathbf{a}_{i}, \mathbf{x}\right\rangle=\mathbf{b}_{i}$.

- Lemma 19. Let $\mathbf{v}_{1}, \ldots, \mathbf{v}_{k} \in \mathbb{R}^{n}$ be linearly independent vectors that satisfy the $\delta$-distance property and let $\pi$ be the orthogonal projection onto the subspace orthogonal to $\mathbf{v}_{k}$. Then $\pi\left(\mathbf{v}_{1}\right), \ldots, \pi\left(\mathbf{v}_{k-1}\right)$ satisfy the $\delta$-distance property.

Proof. See full paper.

This Lemma, which was already used by [12], implies that if $P$ satisfies the local $\delta$-distance property then so does $F_{i}$, where the definition of local $\delta$-distance is understood relative to the affine hull of $F_{i},{ }^{5}$ because the normal vectors of $F_{i}$ arise from orthogonal projections of the normal vectors of $P$.

5 Alternatively, one can apply a rotation and translation so that $F_{i}$ lies in the subspace $\mathbb{R}^{n-1}$ spanned by the first $n-1$ coordinates. The rotation does not affect the $\delta$-distance property, and we can then treat $F_{i}$ as a polytope in $\mathbb{R}^{n-1}$. 
Input: polytope $P=\left\{\mathbf{x} \in \mathbb{R}^{n}: A \mathbf{x} \leq \mathbf{b}\right\}, \delta>0$, feasible basis $B, \mathbf{d} \in \mathbb{R}^{n}$

Output: optimal basis $B \subset[m]$ for $\mathbf{d}$

$\mathbf{c} \leftarrow \sum_{i \in B} \frac{\mathbf{a}_{i}}{\left\|\mathbf{a}_{i}\right\|}, \mathbf{d} \leftarrow 2 \frac{\mathbf{d}}{\|\mathbf{d}\|}$

Sample $X \in \mathbb{R}^{n}$ from the exponential distribution conditioned on $\|X\| \leq 2 n$

Follow segments $[\mathbf{c}, \mathbf{c}+X],[\mathbf{c}+X, \mathbf{d}+X],\left[\mathbf{d}+X, \mathbf{d}+\frac{\delta}{2 n^{3}} X\right]$ using Shadow Simplex

Find $\lambda_{i}$ such that $\tilde{\mathbf{d}}:=\mathbf{d}+\frac{\delta}{2 n^{3}} X=\sum_{i \in B} \lambda_{i} \frac{\mathbf{a}_{i}}{\left\|\mathbf{a}_{i}\right\|}$ where $B$ is the current basis

Choose $i^{\star}$ such that $\lambda_{i^{\star}}>\frac{1}{n}$

$B^{\prime} \leftarrow$ optimal basis of $F_{i^{\star}}$ for $\pi_{i^{\star}}(\mathbf{d})$, obtained by recursion starting at $B \backslash\left\{i^{\star}\right\}$

return $B^{\prime} \cup\left\{i^{\star}\right\}$

Algorithm 1: Optimization

Theorem 20. If $P$ satisfies the local $\delta$-distance property, then Algorithm 1 correctly computes an optimal basis for $\mathbf{d}$ using an expected $O\left(\left(n^{3} / \delta\right) \ln (n / \delta)\right)$ shadow simplex pivots.

Proof. For correctness, let $\mathcal{T}$ be some triangulation of the normal fan of $P$ and let $C$ be a cone in $\mathcal{T}$ that contains $\mathbf{d}$. We have $\left\|\frac{\delta}{2 n^{3}} X\right\| \leq \frac{\delta}{n^{2}}$ and therefore $d(\tilde{\mathbf{d}}, C) \leq d(\tilde{\mathbf{d}}, \mathbf{d}) \leq \frac{\delta}{n^{2}}$. Furthermore, $\|\tilde{\mathbf{d}}\| \geq\|\mathbf{d}\|-\frac{\delta}{n^{2}}>1$ implies that $\sum_{i \in B} \lambda_{i}>1$ so that there is some $i$ with $\lambda_{i}>\frac{1}{n}$. Applying Lemma 18 yields that $\mathbf{a}_{i^{\star}}$ is a generator of $C$, which means that $i^{\star}$ is contained in some optimal basis for $\mathbf{d}$. This implies that recursion on $F_{i^{\star}}$ yields the correct result.

In order to bound the number of pivots, let $C$ be the cone of the initial basis and observe that $\mathbf{c}+\delta \mathcal{B}_{2}^{n} \subseteq C$ by the proof of Lemma 5 . Hence the segment $\left[\mathbf{c}+\frac{\delta}{2 n} X\right)$ does not cross a facet of the triangulation $\mathcal{T}_{1}$ of the normal fan that is implicitly used by the first leg of the shadow simplex path.

If $X$ were exponentially distributed (without the conditioning on $\|X\| \leq 2 n$ ), Theorem 10 together with Lemma 5 would bound the expected number of pivot steps along the three segments by

$$
\mathbb{E}[N] \leq \frac{2 n^{2}}{\delta} \ln \frac{2 n}{\delta}+\frac{n\|\mathbf{d}-\mathbf{c}\|}{\delta}+\frac{2 n^{2}}{\delta} \ln \frac{2 n^{3}}{\delta} \leq O\left(\frac{n^{2}}{\delta} \ln \left(\frac{n}{\delta}\right)\right)
$$

Since $\mathbb{E}[\|X\|]=n$ we have $\operatorname{Pr}[\|X\| \leq 2 n] \geq \frac{1}{2}$ by Markov's inequality and therefore

$$
\mathbb{E}[N \mid\|X\| \leq 2 n] \leq 2 \mathbb{E}[N] \leq O\left(\frac{n^{2}}{\delta} \ln \left(\frac{n}{\delta}\right)\right) .
$$

The bound on the total expected number of pivot steps follows from the depth $n$ of recursion.

\section{Intersection Bounds and Diameter Bounds}

Lemma 21. Let $C$ be a polyhedral cone containing $\mathbf{u}+\tau \mathcal{B}_{2}^{n}$, where $\|\mathbf{u}\|=1$. Let $\mathbf{c}, \mathbf{d} \in \mathbb{R}^{n}$ and let $X \in \mathbb{R}^{n}$ be exponentially distributed on a full dimensional cone $\Sigma \ni \mathbf{u}$. Then the expected number of times the shifted line segment $[\mathbf{c}+X, \mathbf{d}+X]$ hits the boundary of $C$ is at most

$$
\mathbb{E}[|\partial C \cap[\mathbf{c}+X, \mathbf{d}+X]|] \leq \frac{\|\mathbf{d}-\mathbf{c}\|}{\tau} \int_{0}^{1} \int_{(C-((1-\lambda) c+\lambda d)) \cap \Sigma} \zeta_{\Sigma}(\mathbf{x}) \mathrm{d} \mathbf{x} \mathrm{d} \lambda
$$


Proof. Let $F$ be a facet of $C$. Note that with probability 1 , the line segment $[\mathbf{c}+X, \mathbf{d}+X]$ passes through $F$ at most once. By linearity, we see that

$$
\mathbb{E}[|\partial C \cap[\mathbf{c}+X, \mathbf{d}+X]|]=\sum_{F \text { facet of } C} \operatorname{Pr}[(F \cap[\mathbf{c}+X, \mathbf{d}+X]) \neq \emptyset] .
$$

We now bound the crossing probability for any facet $F$.

We first calculate the hitting probability as

$$
\begin{aligned}
\operatorname{Pr}[F \cap[\mathbf{c}+X, \mathbf{d}+X] \neq \emptyset] & =\operatorname{Pr}[X \in-[\mathbf{c}, \mathbf{d}]+F] \\
& =\int_{-[\mathbf{c}, \mathbf{d}]+F} \zeta_{\Sigma}(\mathbf{x}) \mathrm{d} \mathbf{x} \\
& =|\langle\mathbf{n}, \mathbf{d}-\mathbf{c}\rangle| \int_{0}^{1} \int_{F-((1-\lambda) \mathbf{c}+\lambda \mathbf{d})} \zeta_{\Sigma}(\mathbf{x}) \mathrm{dvol}_{n-1}(\mathbf{x}) \mathrm{d} \lambda \\
& \leq\|\mathbf{d}-\mathbf{c}\| \int_{0}^{1} \int_{(F-((1-\lambda) \mathbf{c}+\lambda \mathbf{d})) \cap \Sigma} c_{\Sigma} e^{-\|\mathbf{x}\|} \operatorname{dvol}_{n-1}(\mathbf{x}) \mathrm{d} \lambda
\end{aligned}
$$

where $\mathbf{n} \in \mathbb{R}^{n}$ is a unit normal vector to $F$ and we use $\operatorname{dvol}_{n-1}(\mathbf{x})$ to indicate an integral with respect to the usual $(n-1)$-dimensional measure on the affine hyperplane spanned by the integration domain. Bounding the hitting probability therefore boils down to bounding the measure of a shift of the facet $F$. Letting $h=|\langle\mathbf{n}, \mathbf{u}\rangle| \geq \tau$ (which holds by assumption on $\mathbf{u}$ ), for any shift $\mathbf{t} \in \mathbb{R}^{n}$ we have that

$$
\begin{aligned}
\int_{(F+\mathbf{t}+\operatorname{cone}(\mathbf{u})) \cap \Sigma} e^{-\|\mathbf{x}\|} \mathrm{d} \mathbf{x} & \geq \int_{((F+\mathbf{t}) \cap \Sigma)+\operatorname{cone}(\mathbf{u})} e^{-\|\mathbf{x}\|} \mathrm{d} \mathbf{x} \quad(\text { since } \mathbf{u} \in \Sigma) \\
& =\int_{0}^{\infty} \int_{((F+\mathbf{t}) \cap \Sigma)+\frac{r}{h} \mathbf{u}} e^{-\|\mathbf{x}\|} \operatorname{dvol}_{n-1}(\mathbf{x}) \mathrm{d} r \\
& =\int_{0}^{\infty} \int_{(F+\mathbf{t}) \cap \Sigma} e^{-\left\|\mathbf{x}+\frac{r}{h} \mathbf{u}\right\|} \operatorname{dvol}_{n-1}(\mathbf{x}) \mathrm{d} r \\
& \geq \int_{0}^{\infty} e^{-r / h} \mathrm{~d} r \int_{(F+\mathbf{t}) \cap \Sigma} e^{-\|\mathbf{x}\|} \operatorname{dvol}_{n-1}(\mathbf{x}) \\
& \geq \tau \int_{(F+\mathbf{t}) \cap \Sigma} e^{-\|\mathbf{x}\|} \operatorname{dvol}_{n-1}(\mathbf{x})
\end{aligned}
$$

The lemma now follows by combining (1),(2),(3), using the fact that the $F+$ cone(u) partition the cone $C$ up to sets of measure 0 .

- Lemma 22. Let $\mathcal{T}=\left(C_{1}, \ldots, C_{k}\right)$ be a partition of a cone $\Sigma$ into polyhedral $\tau$-wide cones. Let $\mathbf{c}, \mathbf{d} \in \mathbb{R}^{n}$ and let $X \in \mathbb{R}^{n}$ be exponentially distributed on $\Sigma$. Then the expected number of facets hit by the shifted line segment $[\mathbf{c}+X, \mathbf{d}+X]$ satisfies

$$
\mathbb{E}[|\partial \mathcal{T} \cap[\mathbf{c}+X, \mathbf{d}+X]|] \leq \frac{\|\mathbf{d}-\mathbf{c}\|}{\tau}
$$


Proof. Using Lemma 21, we bound

$$
\begin{aligned}
\mathbb{E}[|\partial \mathcal{T} \cap[\mathbf{c}+X, \mathbf{d}+X]|] & \leq \sum_{i=1}^{k} \mathbb{E}\left[\left|\partial C_{i} \cap[\mathbf{c}+X, \mathbf{d}+X]\right|\right] \\
& \leq \sum_{i=1}^{k} \frac{\|\mathbf{d}-\mathbf{c}\|}{\tau} \int_{0}^{1} \int_{\left(C_{i}-((1-\lambda) \mathbf{c}+\lambda \mathbf{d})\right) \cap \Sigma} \zeta_{\Sigma}(\mathbf{x}) \mathrm{d} \mathbf{x} \mathrm{d} \lambda \\
& \leq \frac{\|\mathbf{d}-\mathbf{c}\|}{\tau} \int_{0}^{1} \int_{\Sigma} \zeta_{\Sigma}(\mathbf{x}) \mathrm{d} \mathbf{x d} \lambda \\
& \leq \frac{\|\mathbf{d}-\mathbf{c}\|}{\tau}
\end{aligned}
$$

as needed.

For the furthermore, note that each intersection is overcounted twice in the summation above, since each facet belongs to exactly two cones in the partition.

We will need the following simple lemma about the exponential distribution.

- Lemma 23. Let $Y$ be exponentially distributed on $\mathbb{R}_{+}$. Then for any $c \in \mathbb{R}, \mathbb{E}[|Y-c|] \geq$ $|c| / 2$.

Proof. See full paper.

While we could choose $\mathbf{c}$ and $\mathbf{d}$ such that $\mathbf{c}+X$ and $\mathbf{d}+X$ lie in the same cones as $\mathbf{c}$ and $\mathbf{d}$ with high probability, and hence no facets are hit by the segments $[\mathbf{c}, \mathbf{c}+X]$ and $[\mathbf{d}, \mathbf{d}+X]$, this would require us to choose $\|\mathbf{d}-\mathbf{c}\|$ quite large. We will show a better way to bound the number of facets that are hit by the segment $[\mathbf{c}, \mathbf{c}+X]$.

Lemma 24. Let $C \subseteq \mathbb{R}^{n}$ be a polyhedral cone containing $\mathbf{u}+\tau \mathcal{B}_{2}^{n}$, where $\|\mathbf{u}\|=1$. Let $\mathbf{c} \in \mathbb{R}^{n}$ and let $X \in \mathbb{R}^{n}$ be exponentially distributed on a cone $\Sigma \ni \mathbf{u}$. Then for every $\alpha \in(0,1)$ we have

$$
\mathbb{E}[|\partial C \cap[\mathbf{c}+\alpha X, \mathbf{c}+X]|] \leq \frac{2}{\tau} \int_{1}^{1 / \alpha} \frac{1}{s} \int_{(C-s \mathbf{c}) \cap \Sigma}\|\mathbf{x}\| \zeta_{\Sigma}(\mathbf{x}) \mathrm{d} \mathbf{x} \mathrm{d} s
$$

Proof. As in the proof of Lemma 21, we will decompose the expectation over the facets of $C$, where we have

$$
\mathbb{E}[|\partial C \cap[\mathbf{c}+\alpha X, \mathbf{c}+X]|]=\sum_{F \text { facet of } C} \operatorname{Pr}[F \cap[\mathbf{c}+\alpha X, \mathbf{c}+X] \neq \emptyset]
$$

Take a facet $F$ of $C$ and let $\mathbf{n}$ denote a unit normal to $F$ pointing in the direction of the cone (i.e., $\langle n, u\rangle>0$ ).

$$
\begin{aligned}
\operatorname{Pr}[F \cap[\mathbf{c}+\alpha X, \mathbf{c}+X] \neq \emptyset] & =\operatorname{Pr}[X \in[1,1 / \alpha](F-\mathbf{c})] \\
& =\int_{1}^{1 / \alpha} \int_{(F-s \mathbf{c}) \cap \Sigma}|\langle\mathbf{n}, \mathbf{c}\rangle| \zeta_{\Sigma}(\mathbf{x}) \mathrm{dvol}_{n-1}(\mathbf{x}) \mathrm{d} s \\
& =\int_{1}^{1 / \alpha} \frac{1}{s} \int_{(F-s \mathbf{c}) \cap \Sigma}|\langle\mathbf{n}, s \mathbf{c}\rangle| c_{\Sigma} e^{-\|\mathbf{x}\|} \operatorname{dvol}_{n-1}(\mathbf{x}) \mathrm{d} s
\end{aligned}
$$


Again, we have to bound an integral over a shifted facet, similar to the proof of Lemma 21. Letting $h=|\langle\mathbf{n}, \mathbf{u}\rangle| \geq \tau$, we have that

$$
\begin{aligned}
& \int_{(F+\mathbf{t}+\operatorname{cone}(\mathbf{u})) \cap \Sigma}\|\mathbf{x}\| e^{-\|\mathbf{x}\|} \mathrm{d} \mathbf{x} \geq \int_{((F+\mathbf{t}) \cap \Sigma)+\operatorname{cone}(\mathbf{u})}\|\mathbf{x}\| e^{-\|\mathbf{x}\|} \mathrm{d} \mathbf{x} \quad(\text { since } \mathbf{u} \in \Sigma) \\
& =\int_{0}^{\infty} \int_{((F+\mathbf{t}) \cap \Sigma)+\frac{r}{h} \mathbf{u}}\|\mathbf{x}\| e^{-\|\mathbf{x}\|} \operatorname{dvol}_{n-1}(\mathbf{x}) \mathrm{d} r \\
& =\int_{0}^{\infty} \int_{(F+\mathbf{t}) \cap \Sigma}\left\|\mathbf{x}+\frac{r}{h} \mathbf{u}\right\| e^{-\left\|\mathbf{x}+\frac{r}{h} \mathbf{u}\right\|} \operatorname{dvol}_{n-1}(\mathbf{x}) \mathrm{d} r \\
& \geq \int_{0}^{\infty} \int_{(F+\mathbf{t}) \cap \Sigma}\left|\left\langle\mathbf{n}, \mathbf{x}+\frac{r}{h} \mathbf{u}\right\rangle\right| e^{-r / h} e^{-\|\mathbf{x}\|} \operatorname{dvol}_{n-1}(\mathbf{x}) \mathrm{d} r \\
& =h^{2} \int_{0}^{\infty}|\langle\mathbf{n}, \mathbf{t}\rangle / h+s| e^{-s} \mathrm{~d} s \int_{(F+\mathbf{t}) \cap \Sigma} e^{-\|\mathbf{x}\|} \operatorname{dvol}_{n-1}(\mathbf{x}) \\
& \geq \frac{h}{2} \int_{(F+\mathbf{t}) \cap \Sigma}|\langle\mathbf{n}, \mathbf{t}\rangle| e^{-\|\mathbf{x}\|} \operatorname{dvol}_{n-1}(\mathbf{x}) \quad \text { (by Lemma 23) } \\
& \geq \frac{\tau}{2} \int_{(F+\mathbf{t}) \cap \Sigma}|\langle\mathbf{n}, \mathbf{t}\rangle| e^{-\|\mathbf{x}\|} \operatorname{dvol}_{n-1}(\mathbf{x})
\end{aligned}
$$

The Lemma now follows by combining (4),(5),(6).

- Lemma 25. Let $\mathcal{T}=\left(C_{1}, \ldots, C_{k}\right)$ be partition of a cone $\Sigma$ into polyhedral $\tau$-wide cones. Let $\mathbf{c} \in \mathbb{R}^{n}$ and $\alpha \in(0,1)$ be fixed and let $X \in \mathbb{R}^{n}$ be exponentially distributed over $\Sigma$. Then

$$
\mathbb{E}[|\partial \mathcal{T} \cap[\mathbf{c}+\alpha X, \mathbf{c}+X]|] \leq \frac{2 n}{\tau} \ln \frac{1}{\alpha} .
$$

Proof. By Lemmas 13 and 24, we have that

$$
\begin{aligned}
\mathbb{E}[|\partial \mathcal{T} \cap[\mathbf{c}+\alpha X, \mathbf{c}+X]|] & \leq \sum_{i=1}^{k} \mathbb{E}\left[\left|\partial C_{i} \cap[\mathbf{c}+\alpha X, \mathbf{c}+X]\right|\right] \\
& \leq \frac{2}{\tau} \sum_{i=1}^{k} \int_{1}^{1 / \alpha} \frac{1}{s} \int_{\left(C_{i}-s \mathbf{c}\right) \cap \Sigma}\|\mathbf{x}\| \zeta_{\Sigma}(\mathbf{x}) \mathrm{d} \mathbf{x} \mathrm{d} s \\
& \leq \frac{2}{\tau} \int_{1}^{1 / \alpha} \frac{1}{s} \int_{\Sigma}\|\mathbf{x}\| \zeta_{\Sigma}(\mathbf{x}) \mathrm{d} \mathbf{x} \mathrm{d} s \\
& \leq \frac{2}{\tau} \int_{1}^{1 / \alpha} \frac{1}{s} \mathbb{E}[\|X\|] \mathrm{d} s=\frac{2 n}{\tau} \ln \frac{1}{\alpha}
\end{aligned}
$$

Acknowledgements. We would like to thank Friedrich Eisenbrand and Santosh Vempala for useful discussions and an anonymous referee for valuable remarks.

\section{References}

1 Karim Alexander Adiprasito and Bruno Benedetti. The Hirsch conjecture holds for normal flag complexes. Arxiv Report 1303.3598, 2014.

2 M. L. Balinski. The Hirsch conjecture for dual transportation polyhedra. Math. Oper. Res., 9(4):629-633, 1984.

3 David Barnette. An upper bound for the diameter of a polytope. Discrete Math., 10:9-13, 1974. 
4 Nicolas Bonifas, Marco Di Summa, Friedrich Eisenbrand, Nicolai Hähnle, and Martin Niemeier. On sub-determinants and the diameter of polyhedra. Discrete Comput. Geom., 52(1):102-115, 2014. Preliminary version in SOCG 12.

5 Karl-Heinz Borgwardt. The simplex method: A probabilistic analysis, volume 1 of Algorithms and Combinatorics: Study and Research Texts. Springer-Verlag, Berlin, 1987.

6 Graham Brightwell, Jan van den Heuvel, and Leen Stougie. A linear bound on the diameter of the transportation polytope. Combinatorica, 26(2):133-139, 2006.

7 Tobias Brunsch and Heiko Röglin. Finding short paths on polytopes by the shadow vertex algorithm. In Automata, languages, and programming. Part I, volume 7965 of Lecture Notes in Comput. Sci., pages 279-290. Springer, Heidelberg, 2013.

8 Daniel Dadush and Nicolas Bonifas. Short paths on the voronoi graph and closest vector problem with preprocessing. In Piotr Indyk, editor, Proceedings of the Twenty-Sixth Annual ACM-SIAM Symposium on Discrete Algorithms, SODA 2015, San Diego, CA, USA, January 4-6, 2015, pages 295-314. SIAM, 2015.

9 Daniel Dadush and Nicolai Hähnle. On the shadow simplex method for curved polyhedra (draft of full paper). Arxiv Report 1412.6705, 2014.

10 Jesús A. De Loera, Edward D. Kim, Shmuel Onn, and Francisco Santos. Graphs of transportation polytopes. J. Combin. Theory Ser. A, 116(8):1306-1325, 2009.

11 Martin Dyer and Alan Frieze. Random walks, totally unimodular matrices, and a randomised dual simplex algorithm. Math. Programming, 64(1, Ser. A):1-16, 1994.

12 Friedrich Eisenbrand and Santosh Vempala. Geometric random edge. Arxiv Report 1404.1568, 2014.

13 Gil Kalai. The diameter of graphs of convex polytopes and $f$-vector theory. In Applied geometry and discrete mathematics, volume 4 of DIMACS Ser. Discrete Math. Theoret. Comput. Sci., pages 387-411. Amer. Math. Soc., Providence, RI, 1991.

14 Gil Kalai and Daniel J. Kleitman. A quasi-polynomial bound for the diameter of graphs of polyhedra. Bull. Amer. Math. Soc. (N.S.), 26(2):315-316, 1992.

15 D. G. Larman. Paths of polytopes. Proc. London Math. Soc. (3), 20:161-178, 1970.

16 Benjamin Matschke, Francisco Santos, and Christophe Weibel. The width of 5-dimensional prismatoids. Arxiv Report 1202.4701, 2013.

17 Denis Naddef. The Hirsch conjecture is true for (0,1)-polytopes. Math. Programming, 45(1, Ser. B):109-110, 1989.

18 Francisco Santos. A counterexample to the Hirsch conjecture. Ann. of Math. (2), 176(1):383-412, 2012.

19 Daniel A. Spielman and Shang-Hua Teng. Smoothed analysis of algorithms: why the simplex algorithm usually takes polynomial time. J. ACM, 51(3):385-463 (electronic), 2004.

20 Michael J. Todd. An improved Kalai-Kleitman bound for the diameter of a polyhedron. Arxiv Report 1402.3579, 2014.

21 Roman Vershynin. Beyond Hirsch conjecture: walks on random polytopes and smoothed complexity of the simplex method. SIAM J. Comput., 39(2):646-678, 2009. 